\title{
Educación viralizada desde la universidad: modelo tecno-pedagógico para trascender los límites del aula en el proceso de aprendizaje
}

Viral learning from the university: a techno-pedagogical model to transcend classroom boundaries in the learning process

Educació viralitzada en la universitat: model tecnopedagògic per a transcendir els límits de l'aula en el procés d'aprenentatge

\author{
Quelic Berga-Carreras*® $^{\circledR}$, Elena Barberà ${ }^{\circledR}$ \\ Universitat Oberta de Catalunya, Barcelona, España \\ *Autor para correspondencia: qberga@uoc.edu (Quelic Berga-Carreras)
}

Recibido: 25/05/2021 | Aceptado: 18/10/2022 | Publicado: 15/01/2022

\begin{abstract}
Cómo citar: Berga-Carreras, Q. y Barberà, E. (2022). Educación viralizada desde la universidad: modelo tecno-pedagógico para trascender los límites del aula en el proceso de aprendizaje. Research in Education and Learning Innovation Archives, 28,1-15.
\end{abstract}

10.7203/realia.28.20977

Copyright: El/La Autor/a. Open Access: Este es un artículo de acceso abierto distribuido bajo los términos de la licencia Creative Commons

Attribution-NoDerivatives 4.0 International licence (CC BY-ND 4.0)

Financiación: None informed
RESUMEN: El aprendizaje universitario tiene vocación de ser utilizado y transferido a otros contextos, sobre todo en el campo profesional. El marco de la educación por competencias es un campo ideado para ello pero se detecta la necesidad de mecanismos que impulsen la comunicación de este conocimiento de manera efectiva y fiable. En este artículo se presenta el proceso de diseño, refinamiento e implementación de un modelo articulado mediante una herramienta inspirada en la filosofía de e-portafolio. Este aporta una capa tecnopedagógica robusta que facilitaría la viralización tutorizada de los conocimientos propios de las disciplinas universitarias que acabarían conformando una red de perfiles y contenidos supervisados por docentes. Todo ello se gesta a partir de la capacitación de los estudiantes, desde la institución, para crear redes de conocimiento activo que rompan el estancamiento formal y la linealidad docente expandiendo el saber, adquirido de manera tutorizada, a una comunidad abierta y más amplia. La metodología utilizada es la Investigación Basada en el Diseño aplicada entre 2015 y 2019. El resultado es una herramienta conceptual capaz de transformar fehacientemente el diálogo entre la universidad y los distintos contextos de aplicación del conocimiento gestado en su propio entorno. En el artículo se describen los constructos desarrollados en el refinamiento del modelo propuesto que está siendo utilizado de manera generalizada en un entorno universitario en línea.

PALABRAS CLAVE: Investigación basada en el diseño; metodologías ágiles; Aprendizaje en red; Sistemas de Gestión de Contenidos; Portafolio

ABSTRACT: University learning is intended to be applied and transferred to other contexts, especially in professional settings. The education-by-competence framework is designed with this in mind. Mechanisms are needed, however, that promote the communication of this knowledge effectively and reliably. This article presents the process for designing, refining and implementing a model based on an instrument inspired by the e-portfolio philosophy. This model provides a robust techno-pedagogical layer intended to facilitate the tutored viralization of knowledge from university disciplines that lead to the creation of a network of teacher-supervised profiles and content. All this output comes from the students of the institutions themselves and their ability to generate active-knowledge networks that break with formal stagnation and teaching linearity by expanding the knowledge acquired in supervised fashion to an open and wider community. The methodology used for this study, conducted between 2015 and 2019, was Design-Based Research. The result is a 
conceptual tool that can reliably generate an open dialogue between the university and the contexts in which university-developed knowledge is applied. This article describes the constructs developed during the model-refinement process. This model is now being used in generalized fashion in an online university environment.

KEYWORDS: Design Based Research; Agile Methodologies; Networked Learning; Content Management Systems; Portfolio

RESUM: L'aprenentatge universitari té vocació de ser utilitzat i transferit a altres contextos, sobretot en el camp professional. El marc de l'educació per competències és un camp ideat per a això, però es detecta la necessitat de disposar de mecanismes que impulsen la comunicació d'aquest coneixement de manera efectiva i fiable. En aquest article es presenta el procés de disseny, refinament i posada en pràctica d'una eina que es basa en els principis -e, que aporta una capa robusta que vol facilitar la tutorització dels coneixements propis de les disciplines universitàries amb el qual poden acabar conformant una xarxa de perfils i continguts supervisats per docents. Tot això a partir de la capacitació dels estudiants en la institució per a crear xarxes de coneixement actiu que trenquen l'estancament formal i la linealitat docent i expandeixen el saber adquirit de manera tutoritzada per una comunitat oberta i més àmplia. La metodologia utilitzada és la investigació basada en el disseny que es va fer entre els anys 2015 i 2019. El resultat és una eina conceptual capaç de transformar a fons el diàleg entre la universitat i els diferents contextos d'aplicació del coneixement que s'obté en el seu propi entorn. En l'article s'exposen els constructes que s'han elaborat per refinar el model que es proposa i que s'està utilitzant de manera general en un entorn universitari en línia.

PARAULES CLAU: Investigació basada en el disseny; metodologies àgils; Aprenentatge en xarxa; Sistemes de Gestió de Continguts; Portafolis

Notas de aplicación práctica

\section{Qué se sabe sobre el tema}

- Los e-portafolios son apropiados para la evaluación por competencias.

- Es un reto para las universidades integrar el aprendizaje informal con el formal.

- Una de las misiones de las universidades es transmitir el conocimiento adquirido en el aula hacia la sociedad y el sector profesional.

\section{Qué aporta este trabajo}

- Los e-portafolios son apropiados para generar red mediante la generación de identidad digital.

- Conseguir integrar los gestores de aprendizaje (LMS) con gestores de contenidos (CMS) libres como WordPress empoderan a la comunidad estudiantil.

\section{Implicaciones para la práctica y/o política}

- Generar una red social académica de conocimiento, listo para extenderse entre instituciones.

- Facilitar el empoderamiento de la comunidad universitaria mediante la capacidad de publicar contenidos propios en la red, supervisados y/o avalados por la institución.

\section{INTRODUCCIÓN Y ESTADO DE LA CUESTIÓN}

A menudo nos lamentamos de que la adquisición de conocimientos en contextos formales no traspasa las paredes de los edificios donde se lleva a cabo. El saber compartido parece ser inerte por estancado y porque se plantea en muchos casos de manera transmisiva, lineal y unilateral de profesor a alumno. Del mismo modo, relacionar el aprendizaje formal con el informal ha sido estudiado como un problema relevante para las universidades (Becker et al., 2017), reto que afecta también la capacidad de conectar el conocimiento adquirido en el aula con la sociedad y el sector profesional (Fung, 2017). En este contexto se manifiestan fuertes dificultades para valorar el grado y la naturaleza de la intersubjetividad que implica un proceso de comprensión mutua y 
significativa. La enseñanza híbrida y en línea han dado alas a esta criticada cerrazón por su propia vocación expansiva pero tampoco han conseguido superar dichas limitaciones. Como respuesta, se busca empoderar al estudiante universitario (y también al profesorado) para crear una red que fracture la linealidad y unilateralidad de la enseñanza actual.

Los sistemas de gestión del aprendizaje, o Learning Management Systems (LMS), perpetúan a menudo el patrón de educación basada en la transmisión (Aparici y Silva, 2012; Laurillard, 2013) donde el personal docente tiene un rol de control y administración de contenidos y tareas, y en el que el estudiante es receptor y ejecutor de estas. Apreciamos en los principales LMS (Canvas, Moodle, Blackboard, Desire2Learn, etc.) como estos patrones se mantienen, siguiendo metáforas y constructos propios de las pedagogías más tradicionales. El uso de analogías del entorno físico, como por ejemplo el uso de la metáfora del alumno que entra en una aula virtual para aprender, nos facilita mucho el modelado de la interacción en un espacio virtual. De todos modos no deberíamos subestimar las posibilidades específicas de los entornos computacionales y las propiedades intrínsecas de las redes (Murray, 2012).

El potencial de lo computacional, junto con las redes, no sólo traslada el proceso de enseñanza-aprendizaje sino que lo sostiene en un entorno de características y posibilidades propias, que desoyen ciertas leyes del entorno físico (Maeda, 2019). Por ejemplo, el aula virtual es una propuesta de representación, una metáfora materialdigital (den Boomen, 2014), que sirve de analogía de las acciones que se organizan en espacios educativos físicos. Cuando estamos estudiando o aprendiendo usando la Red, esos espacios son literalmente representaciones (Echeverría, 1999). Pero de hecho, lo formal e informal, lo cerrado y lo abierto, lo privado y lo social, cuando operamos desde el cristal de nuestros dispositivos, dejan de ser un acto que se celebra en espacios realmente separados. Cuando conceptualizamos un espacio docente en la Red, a menudo caemos en la tentación de marcar ciertas representaciones y separaciones por inercia o tradición, pero en el fondo, se abre la posibilidad de interactuar, relacionarse $\mathrm{y}$, por ende, de enseñar y aprender de maneras más propias del entorno digital.

Las tecnologías de la información y comunicación (TIC) nos han permitido formalizar paradigmas pedagógicos basados en conceptos como la red, lo horizontal, o incluso la conectividad, repensando e incluso superando la idea de la educación como transmisión de conocimiento (Anderson y Dron, 2014; Downes, 2010; Greenhow y Lewin, 2016). En este sentido, hay un creciente volumen de investigaciones que exploran las redes personales de aprendizaje alimentadas constantemente por experiencias entre los contextos formales e informales (Oddone, Hughes, y Lupton, 2019; Peters y Romero, 2019).

Aunque casi el $40 \%$ de los alumnos suplantaría la plataforma de gestión de aprendizaje de la universidad por alguna red social, y estudios recientes demuestran que tanto alumnos como profesores usan redes formales e informales para optimizar su aprendizaje, los LMS no están diseñados para integrar esas experiencias informales (Czerkawski, 2016). En este sentido, el aprovechamiento académico de la introducción masiva de las redes sociales en Europa (de la cual existen valoraciones contrapuestas sobre su uso educativo) aún es tangencial (Gewerc-Barujel, Montero-Mesa, y LamaPenín, 2014).

Existe una fuerte evidencia de que los medios y redes sociales pueden facilitar la agregación y la compartición de procesos y resultados de aprendizaje universitario, la participación en la generación colectiva de conocimiento, la gestión de la propia atribución de sentido a lo aprendido y el trasvase entre lo formal y lo informal (Dabbagh y Kitsantas, 2012; Peters y Romero, 2019).

Se pronostica que la futura generación de las plataformas de gestión del aprendizaje (LMS) deberían incorporar diferentes formas de participación, si quieren mantenerse como opción para encuadrar las propuestas de e-learning (Mott, 2010), pero la realidad 
es que hay pocas iniciativas suficientemente generalizadas y robustas al respecto que consideren el potencial de diseminar y viralizar lo aprendido desde un contexto universitario formal hacia contextos más informales. Entendemos por viralizar el hecho de permitir de forma no controlada la diseminación de contenidos generados por la propia comunidad.

Para lograr esta coherencia integrando lo social con lo pedagógico dentro de entornos de aprendizaje en línea o híbridos, es necesaria una aproximación multidisciplinar, tanto en la práctica como en la investigación pedagógica, que pueda contemplar las especificidades de cada disciplina, generando propuestas entre los ámbitos de las ingenierías y las ciencias sociales (Selwyn et al., 2020).

Manteniendo una mirada multidisciplinar y crítica con las TIC, parece relevante conectar la cultura de lo compartido y de la participación (Jenkins, Clinton, Purushotma, Robison, y Weigel, 2009) desde cualquier emisor y contexto, junto a modelos formales de aprendizaje. Parece interesante potenciar la propagación del aprendizaje formal hacia redes informales (Greenhow y Lewin, 2016) para integrar el potencial de las estructuras universitarias, junto a sus LMS y el de las redes sociales.

Para ello proponemos el desarrollo de un modelo tecno-pedagógico; un modelo de interacción online acompañado de una serie de usos y metodologías para el uso pedagógico, inspirado en el modelo del portafolio electrónico (Barberà, Gewerc-Barujel, y Rodríguez-Illera, 2016), que permite explorar de forma empírica maneras propias de generar, acompañar o incentivar el proceso de aprendizaje a lo largo de la vida, aprovechando el potencial de la computación y la capacidad de diseminar e incluso viralizar contenidos en la Red. En concreto, y queriendo responder a la pregunta de cómo empoderar a los estudiantes universitarios para crear una red abierta de conocimiento activo, validado y adquirido de manera tutorizada, abordamos un modelo de solución tecnopedagógica que se concreta en los siguientes objetivos.

1. Valorar de manera multidisciplinar el estado actual de las herramientas de mediación de procesos y resultados de aprendizaje del alumnado basados principalmente en lo textual y los procesos individuales.

2. Integrar conceptual y prácticamente las necesidades detectadas, haciendo énfasis en sus déficits.

3. Proponer una solución holística a medio y largo plazo en prospectiva estratégica con el campus de la creatividad que permita una comunicación más colectiva y rica en medios audiovisuales.

4. Transferir la solución a entornos de educación en línea y presenciales de diferentes áreas de conocimiento.

\section{MATERIAL Y MÉTODOS}

En coherencia con los objetivos marcados, el desarrollo se llevó a cabo mediante la metodología de investigación basada en el diseño (Benito-Crosetti y Salinas-Ibáñez, 2016). Esta metodología que está muy extendida en el campo educativo y orienta al desarrollo de productos, procesos, programas, herramientas y modelos (Reeves, 2006), busca aportar soluciones prácticas que generan conocimiento mediante principios de diseño que se fundamentan en intervenciones anteriores, conocimiento ya existente y distintos métodos aplicados en las ciencias sociales. Se procura así un contexto de interacción y colaboración práctica entre diferentes participantes implicados (en nuestro caso: profesores, equipo de gestión, especialistas en diseño de interactivos, investigadores, personal técnico y estudiantes) que aportan en los distintos niveles del procedimiento: contexto, definición, prototipo, desarrollo, implementación y valoración. 


\subsection{Contexto y recogida de necesidades}

El desarrollo que presentamos contrastado empíricamente, se inicia con el diseño (2014-2015) y posterior despliegue del Grado de Diseño y Creación Digital (Septiembre 2016) y el diseño (2015-2016) y despliegue del Grado de Artes (Septiembre 2017) de la Universitat Oberta de Catalunya (UOC). Estos grados tenían en el momento de redactar este artículo 1442 y 450 estudiantes respectivamente. Las memorias de los grados, redactadas en colaboración con los estudios de Arte y Humanidades, los estudios de Comunicación y los estudios de Informática, Multimedia y Telecomunicaciones, recogían la necesidad de una solución tecnopedagógica a las demandas representacionales e interactivas.

Entre octubre de 2015 y mayo de 2016, una empresa externa realizó un estudio junto con un grupo de expertos y miembros de la comunidad universitaria para, a partir de la co-creación, idear y conceptualizar herramientas que diesen respuesta a las necesidades recogidas por los claustros de ambos grados.

Posteriormente se redactó un informe del Departamento de Investigación en Aprendizaje a Distancia de la Universidad (eLearn Center) basado en entrevistas semi-estructuradas realizadas a un total de 14 profesores del Grado de Diseño y Creación Digital, el Grado de Artes, el Máster Inter-universitario en Mejora de la Enseñanza Infantil y Primaria, el Máster Universitario de Evaluación y Gestión de la Calidad en la Educación Superior, el Máster Universitario de Formación de Profesorado en Educación Secundaria Obligatoria y Bachillerato, Formación Profesional y Enseñanza de Idiomas, el Máster Universitario de Psicopedagogía y el Diploma de Posgrado Evaluar para Aprender (eLearn Center, 2018).

Dichos documentos, en una primera etapa, informaron al Departamento de Tecnología, que optó por contratar un proveedor de portafolio externo, MyDocumenta. En septiembre de 2017 se empezó a usar, con un nivel de aceptación bajo. En una encuesta interna realizada a 79 estudiantes de los grados implicados, sólo el 44,2\% valoraban su satisfacción como buena, alta o muy alta, aunque el 70,9\% consideraban imprescindible tener una herramienta de portafolio. Después de múltiples intentos de adaptación y mejora de la herramienta se optó por el desarrollo de una herramienta propia.

De la documentación recogida y del uso de MyDocumenta, se extrajeron conclusiones relevantes para la contextualización y definición de lo que será la prueba de concepto Folio previo a su posterior desarrollo, implementación y valoración (Berga, Blasco, Melenchón, y Bertan, 2018).

En esta primera etapa (2014-2018) se detectan las necesidades de disponer de un conjunto de herramientas: una galería visual, un portafolio de estudiante, visual y compartido, un dossier personal de aprendizaje, un sistema de evaluación competencial y finalmente un portafolio profesional (eLearn Center, 2018).

Dada la complejidad del proceso de recogida y desarrollo de la herramienta propuesta, se presenta a continuación un resumen cronológico de sus fases para su mejor comprensión (Gráfico 1).

Así pues, de las necesidades recogidas (objetivo A) afloran diversos patrones. Así, se observa (eLearn Center, 2018):

1. De las 25 experiencias, 14 se centran exclusivamente en la unidad semestre sin poder proyectarse más allá y sólo en la parte de portafolio profesional aparecen usos más prolongados. En 5 casos se detecta una transversalidad de corta distancia que alcanza la unidad programa de estudio. La unidad temporal de docencia y gestión universitarias está pensada en el marco de un semestre mientras que una herramienta integrada como la que se contempla precisa un alcance y temporalidad definidas para una vigencia a largo plazo y, sobre todo, sin disrupciones marcadas por comienzos y finales de curso. La herramienta final ha de construirse a través de los semestres y estar accesible constantemente por diferentes agentes 


\section{Proceso de investigación y diseño de Folio}

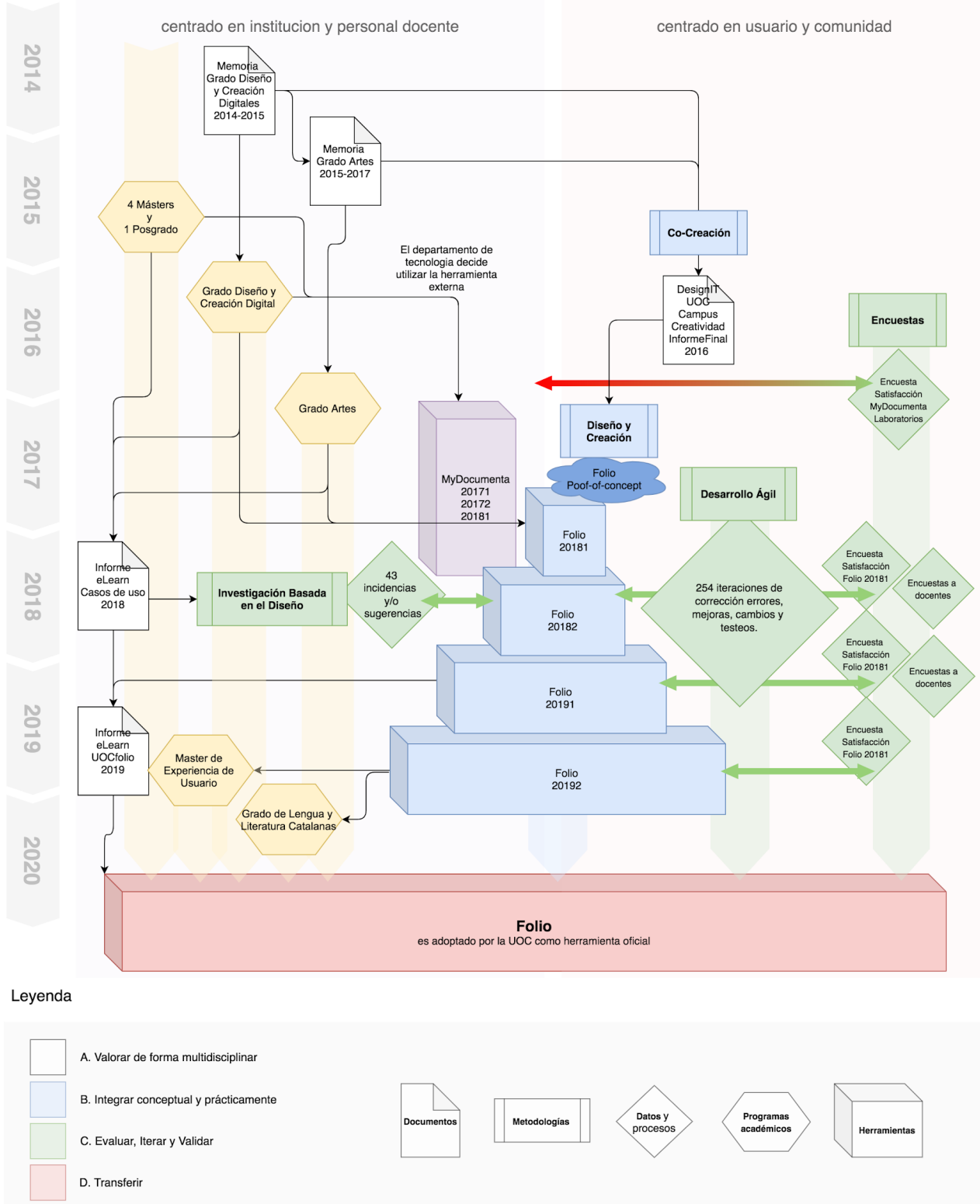

Gráfico 1: Diagrama de investigación y diseño de Folio.

educativos, como mínimo, por profesores y estudiantes pero por su vocación viral también en la red y con sus contenidos localizables en cualquier buscador.

2. Una concordancia de la preferencia entre herramientas y áreas de conocimiento. En cuanto al tipo de herramientas elegidas, entre las cinco experimentadas se muestra una clara preferencia según sea el área de conocimiento. El profesorado de los programas del área de arte y diseño muestra preferencia por el uso de herramientas externas (exceptuando la galería) orientadas a publicar y compartir contenidos. Por su parte, el profesorado de programas del área de educación, muestra preferencia por la herramienta del dossier personal de aprendizaje, diseñada para llevar a cabo procesos de evaluación de los aprendizajes. 
3. Una similitud entre el tipo de finalidad y las áreas de conocimiento que utilizan la herramienta. Se plantean dos finalidades para las herramientas analizadas: evaluativa y expositiva. En relación con lo apuntado en el apartado anterior, 18 de 25 de las experiencias analizadas se dirigen a un fin expositivo mientras que el resto son evaluativas y se encuentran mayoritariamente concentradas en el área de educación ( $66 \%$ de las 7 totales).

4. Una tendencia a la hibridación. Se entiende por hibridación la cohabitación de usos y finalidades diferentes en una misma herramienta constituyendo un sistema más complejo.

\subsection{Instrumentos}

Para responder a las necesidades recogidas en la primera etapa se crea una prueba de concepto entre Marzo y Septiembre de 2018. Esta se valida desde el departamento especializado en e-Learning, conversando con pedagogos expertos en e-portafolios y desarrolladores de EduTech que ponen en valor su potencial (eLearn Center, 2019). Se publican los resultados en un póster (Berga et al., 2018).

Se procede a la creación de un prototipo, y al desarrollo, implementación y valoración de una herramienta de forma iterativa, usando metodologías ágiles (Beck et al., 2001; Hidalgo, 2019), y siguiendo los principios de diseño y creación (March y Smith, 1995; Oates, 2006) que son coherentes con los principios de la Investigación Basada en el Diseño, y la Investigación Científica basada en el Diseño (Design Science Research) usada normalmente en los ámbitos de la ingeniería de software.

Mediante el uso de metodologías ágiles, se aplica un refinamiento continuo que lleva a la mejora del proceso y producto educativo, manteniendo un grupo de usuarios clave informados (profesores, alumnos, técnicos e investigadores) para recoger riesgos y funcionalidades de forma iterativa. Se desarrolla la herramienta a partir de la prueba de concepto. Se establecen dos modos de iteración: un sistema de recogida de modificaciones y un sistema de recogida de incidencias por parte de los usuarios. En concreto, los desarrolladores (técnicos e investigadores) planifican 241 mejoras o resolución de errores de los cuales 194 se resuelven. Por parte de los usuarios (profesores y alumnos) se recogen 43 incidencias o propuestas de mejora de las cuales 39 se resuelven e integran en el proyecto (Gráfico 2). En total se realizan 233 mejoras, implementadas y evaluadas por el equipo de desarrolladores.

\subsection{Participantes y valoración}

El piloto se realiza para un total de 595 participantes a lo largo de tres semestres (Tabla 1).

Para comprobar la utilidad del modelo y la herramienta, se realizan encuestas de satisfacción a los distintos grupos de estudiantes con un total de 309 respuestas sobre 595 participantes $(52 \%)$. Las encuestas dan resultados favorables a la implementación (con valoración global de satisfacción entre buena, alta o muy alta con valores de $71,4 \%$, $71,6 \%$ y $77,1 \%$ en el campus iberoamericano y del $83,3 \%, 66,6 \%$ y $83,9 \%$ en el campus catalán respectivamente). También se realizan encuestas a los profesores durante los dos primeros semestres ( 3 y 8 participantes) para detectar potencialidades y riesgos en el modelo.

\section{ANÁLISIS Y RESULTADOS}

\subsection{Prueba de concepto UOCfolio}

Para integrar conceptual y prácticamente las necesidades detectadas (B) y proponer una solución aplicada (C) se genera una primera prueba de concepto inicialmente 


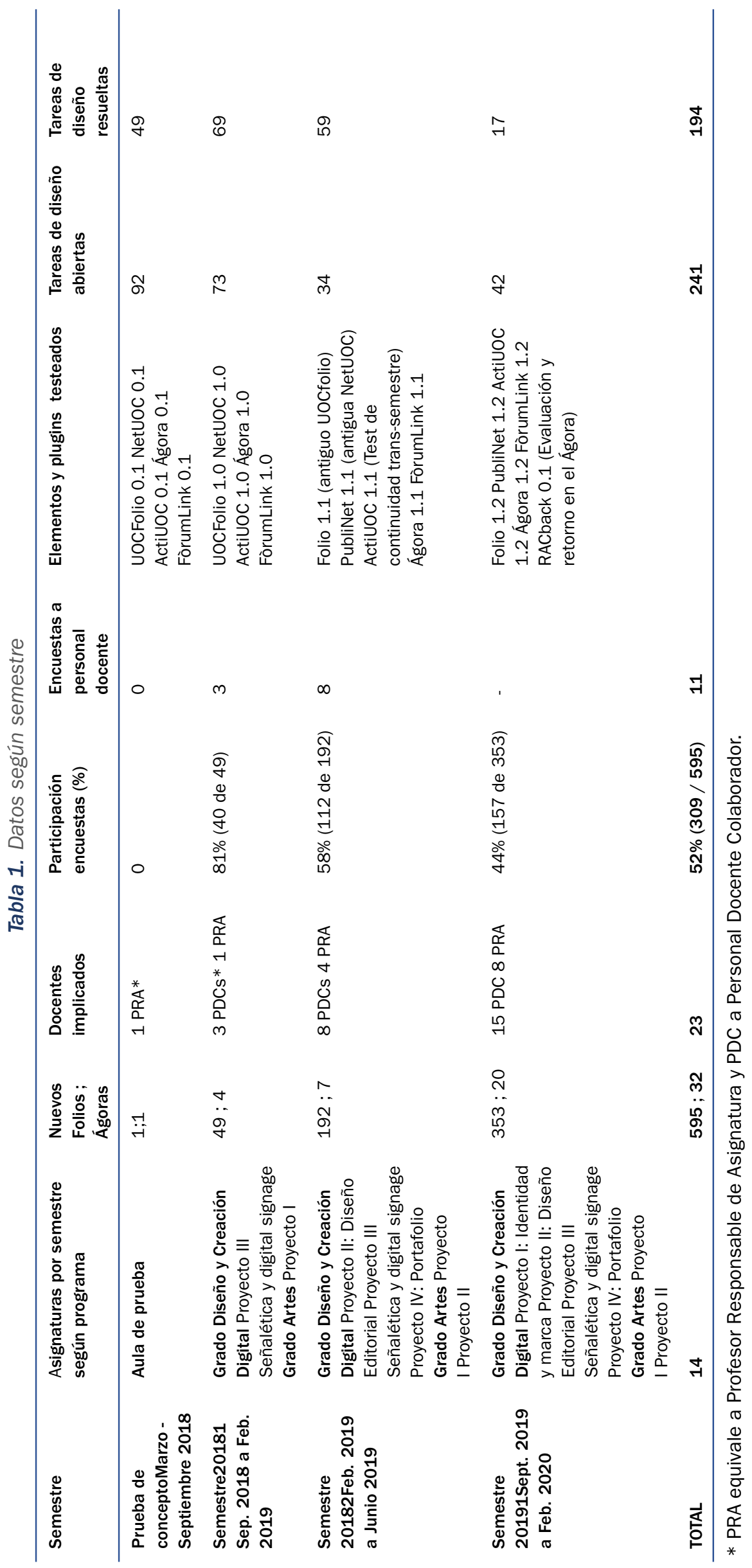



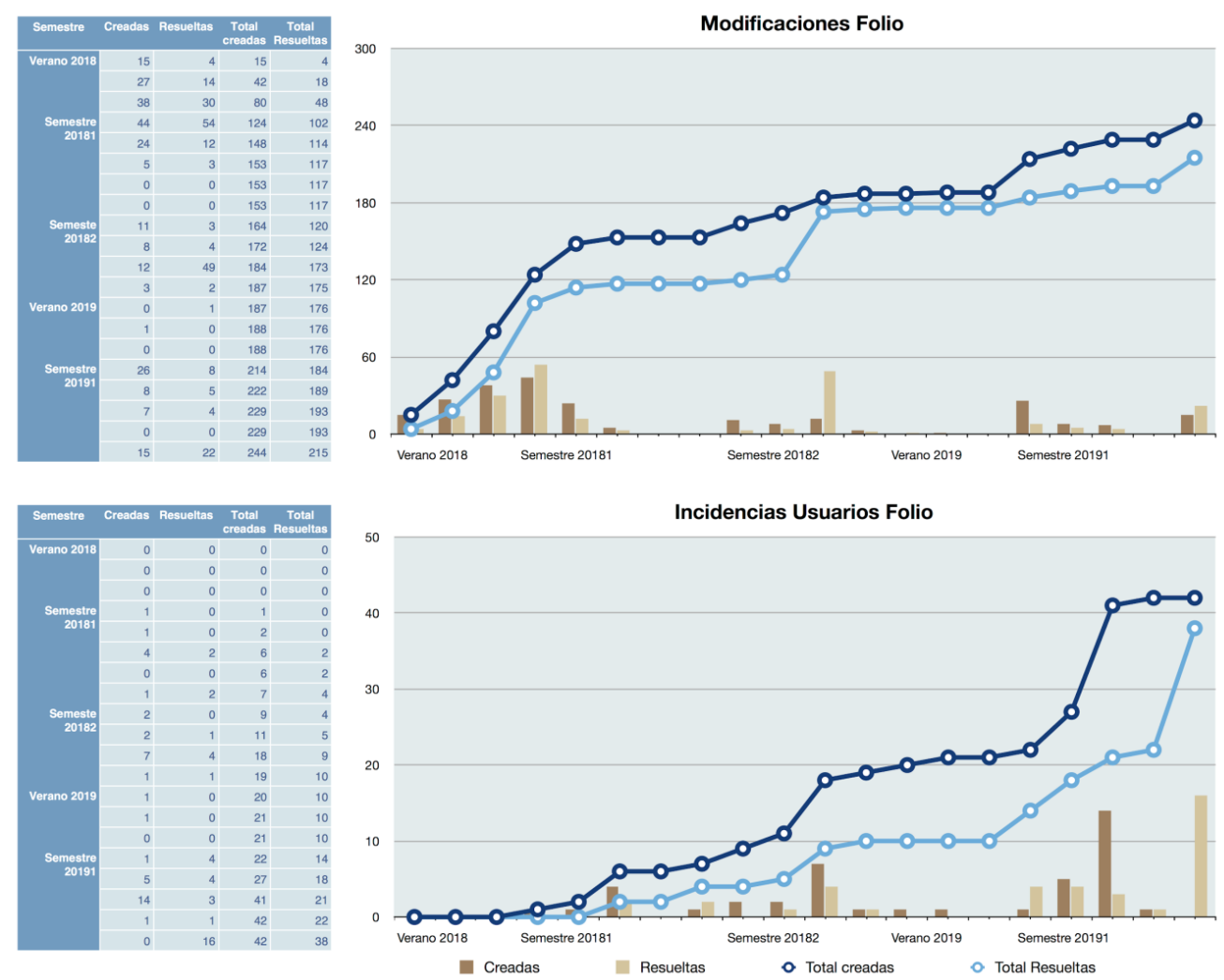

Gráfico 2: Número de modificaciones e iteraciones creadas y resueltas (superior). Incidencias entradas por usuarios y resueltas (inferior).

llamada UOCfolio. El modelo tecno-pedagógico plantea conectar el sistema de gestión de aprendizaje (LMS) de una universidad a distancia con el sistema de gestión de contenidos WordPress (CMS) mediante el uso de las interfaces de programación de aplicaciones (Aplication Programing Interfaces, comúnmente llamadas APIs). Cada estudiante tiene su propio espacio y este, mediante plugins y componentes, puede trabajar con las tareas del LMS. Se opta por WordPress por ser una reputada herramienta de software libre que permite ampliar sus funcionalidades mediante un sistema modular de plugins. Es una elección estratégica que permite aprovechar el potencial de ambos ecosistemas (LMS y CMS) minimizando el desarrollo y garantizando la seguridad de ambos. Otro factor determinante en la elección de WordPress es su abundante y persistente presencia en la red, especialmente en el sector de las artes y el diseño para crear portafolios profesionales.

Se desarrollan cinco modificaciones que combinadas dan cabida inicialmente a tres de las necesidades detectadas (eLearn Center, 2018) mediante una sola herramientamodelo: portafolio de estudiante visual y compartido, dossier personal de aprendizaje, y, portafolio profesional, dejando fuera la evaluación competencial y la galería visual. Esta propuesta, cumpliendo con la tendencia de hibridación, resuelve 1) compartir trabajos 2) evidenciar competencias (aunque inicialmente no evaluarlas) y 3) obtener resultados que sirvan como transición hacia el mundo profesional:

1. UOCfolio: espacio personal del estudiante (WordPress) que puede usar a lo largo de la carrera y de forma transversal entre asignaturas (Gráfico 3 a).

2. NetUOC: sistema de publicación que permite elegir cómo compartir los contenidos: visibilidad pública, campus, aula, personal docente o privada (Gráfico 3 b). 
3. ActiUOC: muestra en la interfaz de edición de WordPress las tareas del LMS pertenecientes a las asignaturas del estudiante, permitiendo así asociar los contenidos del UOCfolio a las actividades docentes (Gráfico 3 c).

4. Ágora: espacio web que recoge los trabajos con permiso de visibilidad aula (NetUOC) pertenecientes a las actividades del grupo de una asignatura (ActiUOC), generando así un sitio común donde ver y compartir los trabajos con los compañeros de estudio.

5. FòrumLink: conector que permite crear y vincular comentarios del CMS a las aulas del LMS. Se conectan así las Ágoras y Folios con los espacios de debate de las aulas.

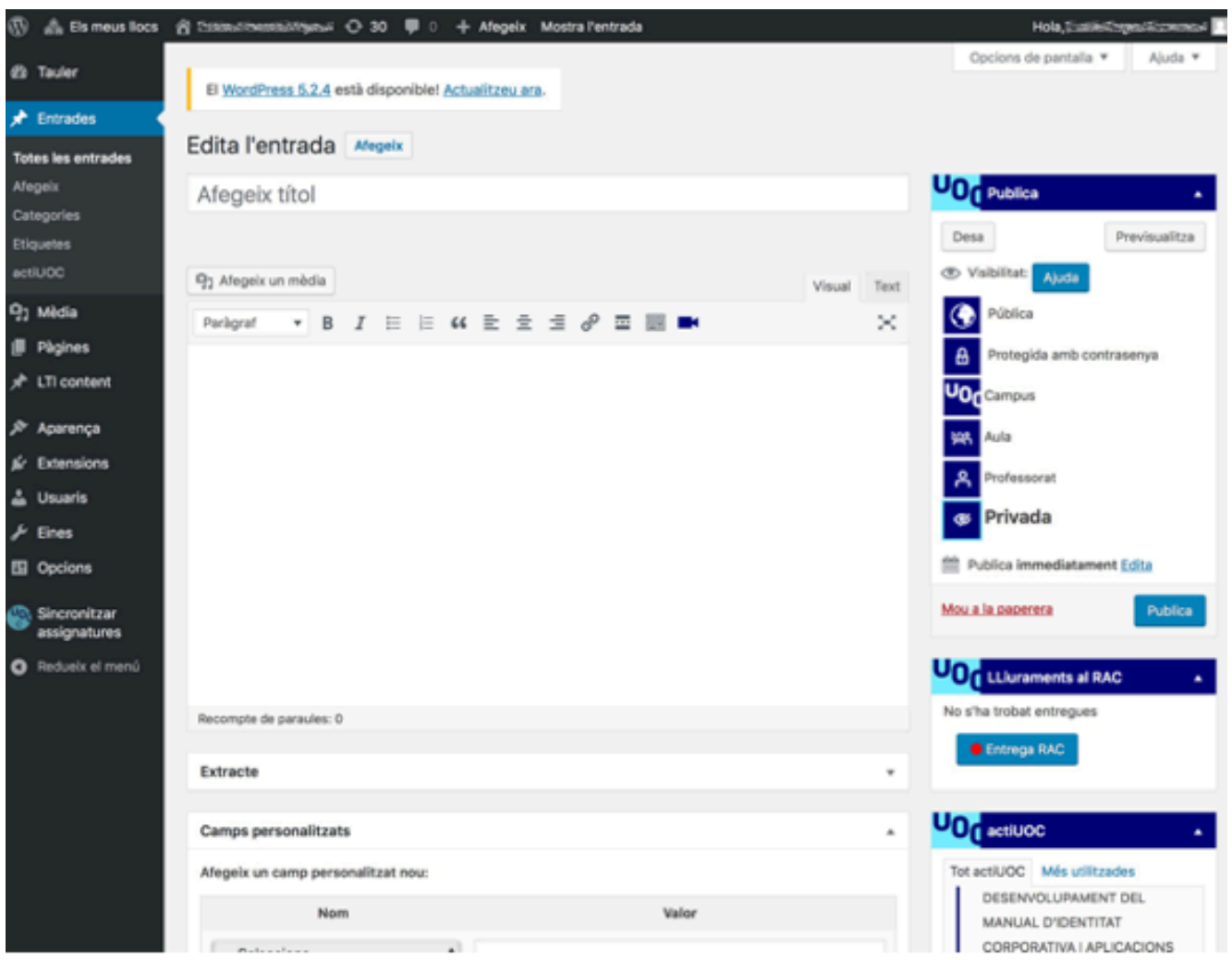

a) UOCfolio: Interfaz de WordPress para la creación de contenidos con los plugins de Folio.

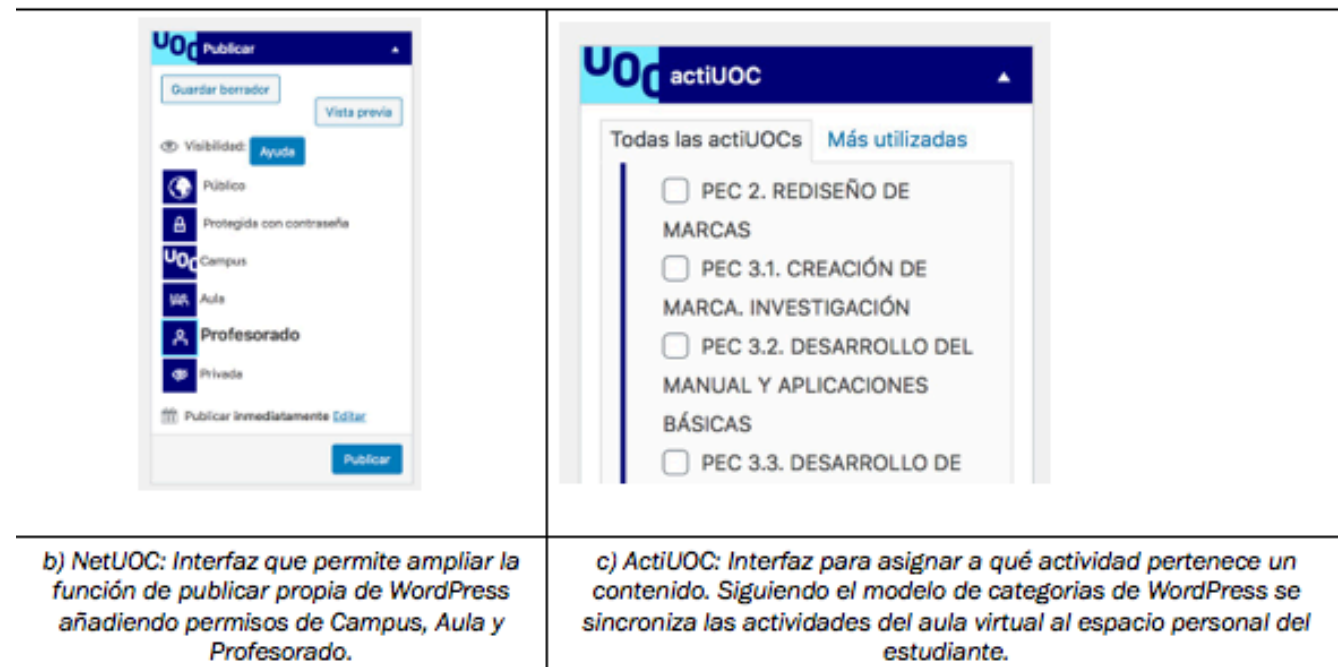

Gráfico 3: Capturas de pantalla de algunos de los desarrollos implementados. 


\subsection{Primer piloto (septiembre 2018)}

Esta prueba inicial se testea en dos asignaturas con un total de 4 profesores y 49 alumnos. Se pone en funcionamiento por primera vez una versión estable del modelo y sus métodos en un entorno real. El equipo técnico aplica 69 modificaciones o correcciones. El $81 \%$ de participantes contestan una encuesta de satisfacción con un alto nivel de satisfacción. El 85\% visita los espacios personales de compañeros, uno de cada dos modifica y adapta algún aspecto de su espacio personal y más del $60 \%$ considera la herramienta como útil o muy útil. Entre los 40 comentarios recogidos, destacamos el interés que despierta la herramienta para compartir con los compañeros por su versatilidad y su potencial hacia el sector profesional:

El uso de UOCfolio creo que es necesario en una carrera que tiene mucha carga práctica y que contiene mucho material visual, como es el Grado en Artes. Es interesante poder ver el desarrollo de los proyectos de compañeros, como si fuese una aula. Estaría bien que fuese equiparable a una web de uso profesional, con toda la libertad que ello conlleva.

\subsection{Segundo Piloto (Febrero 2019)}

Se extiende a 192 estudiantes y 7 aulas, de cinco asignaturas. Se pone especial atención al funcionamiento de las ActiUOCs al cambiar de semestre, ya que los LMS funcionan con asignaturas semestrales y el CMS es a lo largo de la vida y por tanto es importante validar la capacidad trans-semestre del modelo. Se mejora el diseño gráfico de las Ágoras para, por ejemplo, indicar claramente el semestre y aula a la que pertenecen. Se hacen 39 ajustes y se recogen 112 encuestas más. El 55\% utiliza la visibilidad campus sin que se requiera en las asignaturas y el $76,1 \%$ considera interesante poder publicar haciendo el contenido visible en todo el campus. Un $41,2 \%$ hace público alguno de sus contenidos para la red y el 59,7\% valora la opción de publicar en abierto como interesante. Sólo el 8,8\% usa UOCfolio para publicar algo en privado. Comentan: "Ha afectado positivamente en general, ya que el poder ver otras opciones de enfoques de los trabajos de compañeros, facilitan el aprendizaje.", y también, "Creo que es un sistema que se debe conocer y ahondar más en él, ya que actualmente podría ser una herramienta indispensable para el mercado laboral."

El 63,2\% valora positivamente seguir usando la herramienta en siguientes asignaturas. Y, en el caso de las entrevistas al personal docente la valoración positiva es unánime en los 8 entrevistados. Comentan: "El principal beneficio para los alumnos ha sido en relación con la posibilidad de compartir el trabajo con sus compañeros, con el consiguiente incremento de la autoestima y sensación de valor público de su trabajo."

En Junio se emite un nuevo informe (eLearn Center, 2019) que recoge la idoneidad del modelo UOCfolio como solución a las necesidades institucionales. Surge la propuesta de evolucionar el piloto hacia la idea de un escritorio virtual que pueda dar salida a todos los aspectos mencionados en los informes anteriores.

El departamento de marca de la universidad dictamina que el nombre no debe incluir el nombre de la universidad y UOCfolio pasa a llamarse Folio y el plugin de NetUOC pasa a llamarse PubliNET.

\subsection{Tercer Piloto (septiembre 2019)}

Se desarrolla un conector que permite desde el Ágora dar feedback evaluativo y entrar las notas en el Registro de Evaluación del LMS. Este conector, llamado RACback, concilia el interés del profesorado de arte y diseño de que la herramienta sea expositiva y el interés del profesorado del ámbito de educación de usar un dossier personal para integrar el proceso evaluativo dentro de la plataforma. 
Este piloto se realiza con 353 estudiantes y 20 aulas coordinadas por 15 profesores. Se aplican 17 iteraciones de mejora. Contestan las encuestas 157 estudiantes (44\%). Comentan: "Hemos tenido plena visión del trabajo de los compañeros, pudiéndolos contrastar con los nuestros retro-alimentándonos los unos de los otros. Lo que bajo mi punto de vista enriquece nuestro aprendizaje."

\subsection{Modelo de trabajo y difusión de Folio}

Se define un modelo robusto, que pone al estudiante en el centro del proceso de aprendizaje gracias a su escritorio personal (WordPress + modificaciones Folio). Mediante el modelo tecno-pedagógico y su consiguiente ecosistema social de enseñanzaaprendizaje, el estudiante es acompañado a lo largo de la creación de su portafolio académico y profesional. Las encuestas descubren el interés del estudiante en tener su espacio personal más allá de que sea profesional, universitario o propio. Destaca su curiosidad por acceder y consultar los espacios de sus iguales, en las aulas o fuera de ellas. El resultado del diseño e implementación de los citados plugins apuntan hacia una plataforma social que permite la identificación del estudiante con su proceso de aprendizaje formal y que suma, como hemos visto en algunos comentarios, el factor social y de difusión viral a la ecuación del aprendizaje a distancia (Gráfico 4). A los cinco constructos descritos en la prueba de concepto se añaden nuevos usos que surgen de las interacciones en los pilotos y de la experiencia de los usuarios:

1. Diario $\mathbf{P}$ ersonal, propio y privado donde el aprendiz crea o sube sus contenidos manteniendo contenidos en modo borrador o guardados de forma privada.

2. Dossier Personal de Aprendizaje o cuaderno de entregas, cuando se usa con permiso de visibilidad para docentes, y dónde se evalúa mediante el conector RACback. Permite a los docentes ver la trayectoria del estudiante y su historial académico más allá del período de docencia que este imparte.

3. Las Ágoras como espacios web colindantes con el Aula, entendidos como una web a modo de plafón interactivo donde los estudiantes pueden exponer para sus iguales, abriéndose así al debate y al aprendizaje por mímesis.

4. Portafolio del Estudiante que supera los límites del aula para extenderse a todo el campus. Se invita a la socialización y a la identificación personal del estudiante con el resto de la comunidad universitaria.

5. Portafoli o Profesional que recoge los trabajos hechos públicos. Estos contenidos, como valor añadido, son fruto del aval, la tutorización y la evaluación por parte del personal docente.

En total, nueve metáforas material-digitales, cuatro conceptualizadas como plugins que hacen de puente entre el espacio personal y el institucional (ActiUOC, PubliNet, RACback, FòrumLink) y cinco conceptualizadas como configuraciones de espacios que se derivan del uso de los anteriores, se relacionan para formar parte de un modelo que representamos en el Gráfico 4.

Como se observa en el diagrama, una sola herramienta (CMS) tiene distintas funcionalidades y públicos a los que alcanzar, permitiendo extender el proceso de aprendizaje más allá de la relación con el profesorado, el aula o el campus.

Se propone así un modelo de divulgación de contenidos validado en la universidad con capacidad de ser diseminados por la red, logrando así un punto de encuentro entre espacios de la universidad, ámbitos sociales y el sector profesional. Este proyecto se ha convertido estratégicamente en objetivo metodológico institucional por su potencial de expansión del conocimiento compartido, sobre todo en relación a las aportaciones de los estudiantes que durante muchos años se ha mantenido cautivo en las aulas 


\section{Modelo de trabajo y difusión de Folio}

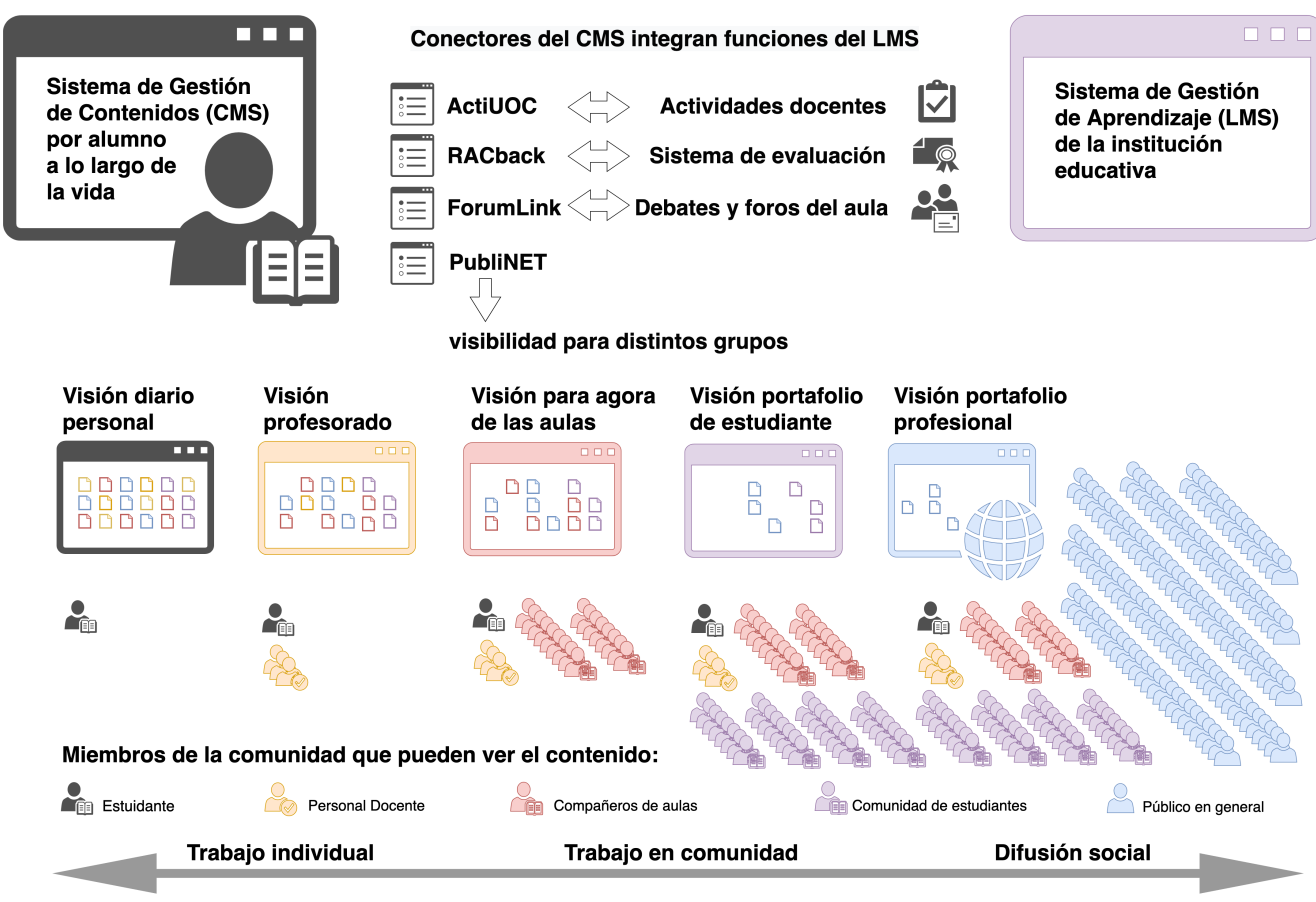

Gráfico 4: Modelo de trabajo y difusión de Folio.

virtuales. Actualmente está aumentando el uso de Folio en prácticamente todos los estudios de la universidad.

La decisión estratégica de apostar por un desarrollo abierto y modular de plugins permite dar solución a futuras necesidades específicas sin romper la consistencia del modelo propuesto. Como fruto de este proceso se posibilita la expansión y transferencia (D) hacia entornos de educación en línea y presenciales de diferentes áreas de conocimiento. El modelo tecno-pedagógico, diseñado para ser compatible con cualquier LMS, facilita su uso por parte de otras instituciones interesadas. Actualmente se están adaptando los plugins para los LMS Moodle y Canvas, facilitando así la transferencia a un número importante de instituciones educativas tanto de Europa como a nivel internacional.

\section{DISCUSIÓN Y CONCLUSIONES}

En este artículo se plantea una solución robusta como respuesta a la necesidad de socializar y conectar el conocimiento universitario mediante la diversificación y la descentralización de la enseñanza. Folio como modelo tecno-pedagógico apodera al estudiante universitario, con la ayuda y validación del personal docente, por medio de la creación de una red compartida de aprendizaje entre iguales. Una solución que suscribe los paradigmas pedagógicos de conectividad, proponiendo un modelo más horizontal, en red. El flujo que propone el modelo recorre un trayecto menos lineal, que desactiva la transmisión unilateral, ya que sustituye metáforas como la del aula presencial (paredes de una clase, caja delimitadora de contenidos) por metáforas de divulgación más orgánicas que facilitan de manera efectiva la transmisión de contenidos y evidencias validadas de aprendizaje (espacio personal, publicación en abierto, compartir con grupos y comunidad). Se proponen constructos que propician la idea de nodos individuales que crean unidades de transmisión y construcción educativa (contenidos, materiales, actividades,...) avalados por la universidad y que conforman 
una red institucional que sobrepasa sus propios límites. Se presenta una vocación de influencia cultural veraz de impacto en internet. Puesto que esta capacidad de divulgar lo aprendido no está exenta de limitaciones, en este artículo se ha querido mostrar no tanto su producto final como el detalle de los procesos implicados en el desarrollo del modelo en sí mismo, para su posible réplica, también requiriendo su evaluación por parte de la comunidad educativa a la que nos mostramos abiertos.

El modelo tecno-pedagógico presentado aporta la creación colectiva de contenidos por parte de los participantes (conceptualizados como personas con agencia completa, y no sólo como estudiantes), los cuales pasan por un proceso de indagación, reelaboración y desarrollo de los contenidos que contribuye a crear un material exportable y supervisado que genera nuevos contenidos. El encadenado de estos procesos, tanto cognitivos como sociales, acaba tejiendo un entramado de nuevo conocimiento generador y utilizable en posteriores nodos de la red ya viralizada.

\section{REFERENCIAS}

Anderson, T., y Dron, J. (2014). Teaching Crowds: Learning and Social Media. Canadá: Athabasca University Press.

Aparici, R., y Silva, M. (2012). Pedagogy of Interactivity. Comunicar, 19(38), 51-58. Descargado de https://doi.org/10.3916/C38-2012-02-05

Barberà, E., Gewerc-Barujel, A., y Rodríguez-Illera, J. L. (2016). Portafolios electrónicos y educación superior en España: Situación y tendencias. Revista de Educación a Distancia (RED), 50. Descargado de https://revistas.um.es/red/article/view/271151

Beck, K., Beedle, M., Van Bennekum, A., Cockburn, A., Cunningham, W., y Fowler, M. (2001). Descargado de https://bit.ly/3aB2M79

Becker, S. A., Cummins, M., Davis, A., Freeman, A., Hall, C. G., y Ananthanarayanan, V. (2017). NMC horizon report: 2017 higher education edition. The New Media Consortium. Descargado de https://library.educause.edu/resources/2017/2/2017-horizon-report

Benito-Crosetti, B. D., y Salinas-Ibáñez, J. M. (2016). La Investigación Basada en Diseño en Tecnología Educativa. Revista Interuniversitaria de Investigación en Tecnología Educativa. http://dx.doi.org/10.6018/riite/2016/260631

Berga, Q., Blasco, L., Melenchón, J., y Bertan, A. (2018). Network Learning Environments: Integrating An Online Learning Model With The Wordpress Publishing Model For The Teaching-learning Of Graphic Design And Arts. Proceedings of the 10th European Distance and E-Learning Network Research Workshop (pp. 392-397).

Czerkawski, B. (2016). Blending Formal and Informal Learning Networks for Online Learning. The International Review of Research in Open and Distributed Learning, 17(3). https://doi.org/10.19173/irrodl.v17i3.2344

Dabbagh, N., y Kitsantas, A. (2012). Personal Learning Environments, social media, and selfregulated learning: A natural formula for connecting formal and informal learning. The Internet and Higher Education, 15(1), 3-8. https://doi.org/10.1016/j.iheduc.2011.06.002

den Boomen, M. V. (2014). Transcoding the digital: How metaphors matter in new media. Institute of Network Cultures.

Downes, S. (2010). Learning Networks and Connective. En H. H. Yang y S. C.-Y. Yuen (Eds.), Collective intelligence and e-learning 2.0: Implications of web-based communities and networking (pp. 1-26). Hershey, PA: Information Science Reference.

Echeverría, J. (1999). Los señores del aire: Telépolis y el tercer entorno. Barcelona: Ediciones Destino.

eLearn Center. (2018). Estudi sobre els usos docents del portafolis i eines visuals a la UOC. Barcelona: Universitat Oberta de Catalunya.

eLearn Center. (2019). Usos docents del UOCFolio en base a l'informe de resultats de l'estudi sobre els usos docents del portafolis i eines visuals a la UOC. Barcelona: Universitat Oberta de Catalunya. 
Fung, D. (2017). A connected curriculum for higher education. London: UCL Press. Descargado de https://discovery.ucl.ac.uk/id/eprint/1558776/1/A-Connected-Curriculum-for -Higher-Education.pdf

Gewerc-Barujel, A., Montero-Mesa, L., y Lama-Penín, M. (2014). Collaboration and Social Networking in Higher Education. Comunicar, 21(42), 55-63. https://doi.org/10.3916/ C42-2014-05

Greenhow, C., y Lewin, C. (2016). Social media and education: Reconceptualizing the boundaries of formal and informal learning. Learning, Media and Technology, 41(1), 6-30. https://doi.org/10.1080/17439884.2015.1064954

Hidalgo, E. S. (2019). Adapting the scrum framework for agile project management in science: Case study of a distributed research initiative. Heliyon, 5(3). https://doi.org/ 10.1016/j.heliyon.2019.e01447

Jenkins, H., Clinton, K., Purushotma, R., Robison, A. J., y Weigel, M. (2009). Confronting the challenges of participatory culture: Media education for the 21st century. Chicago, IL: MIT Press. Descargado de https://www.macfound.org/media/article_pdfs/jenkins _white_paper.pdf

Laurillard, D. (2013). Rethinking university teaching: A conversational framework for the effective use of learning technologies. London: Routledge.

Maeda, J. (2019). How to speak machine: Computational thinking for the rest of us. New York: Portfolio//Penguin.

March, S. T., y Smith, G. F. (1995). Design and natural science research on information technology. Decision Support Systems. Decision Support Systems, 15, 251-266. https://doi.org/10.1016/0167-9236(94)00041-2

Mott, J. (2010). Envisioning the post-LMS era: The open learning network. Educause Quarterly(1), 33-33. Descargado de https://er.educause.edu/articles/2010/3/envisioning -the-postlms-era-the-open-learning-network

Murray, J. H. (2012). Inventing the medium: Principles of interaction design as a cultural practice. Cambridge, Mass: MIT Press.

Oates, B. J. (2006). Researching information systems and computing. London / Thousand Oaks, Calif: SAGE Publications.

Oddone, K., Hughes, H., y Lupton, M. (2019). Teachers as Connected Professionals. The International Review of Research in Open and Distributed Learning, 20(3). https://doi .org/10.19173/irrodl.v20i4.4082

Peters, M., y Romero, M. (2019). Lifelong learning ecologies in online higher education: Students' engagement in the continuum between formal and informal learning. British Journal of Educational Technology, 50(4), 1729-1743.

Reeves, T. (2006). Design research from a technology perspective. Educational design research (pp. 64-78). London: Routledge.

Selwyn, N., Hillman, T., Eynon, R., Ferreira, G., Knox, J., Macgilchrist, F., y Sancho-Gil, J. M. (2020). What's next for Ed-Tech? Critical hopes and concerns for the 2020s. Learning, Media and Technology, 45, 1-6. https://doi.org/10.1080/17439884.2020.1694945 